\title{
Poor outcomes in patients with cirrhosis and Corona Virus Disease-19
}

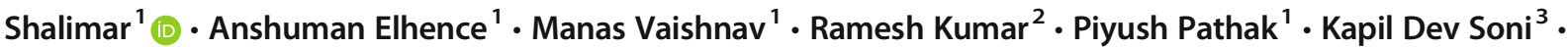 \\ Richa Aggarwal ${ }^{3} \cdot$ Manish Soneja $^{4} \cdot$ Pankaj Jorwal $^{4}$ - Arvind Kumar ${ }^{4} \cdot$ Puneet Khanna $^{3} \cdot$ Akhil Kant Singh $^{3}$. \\ Ashutosh Biswas ${ }^{4} \cdot$ Neeraj Nischal $^{4} \cdot$ Lalit Dar $^{5} \cdot$ Aashish Choudhary $^{5} \cdot$ Krithika Rangarajan $^{6} \cdot$ Anant Mohan $^{7}$. \\ Pragyan Acharya $^{8}$ - Baibaswata Nayak ${ }^{1}$. Deepak Gunjan ${ }^{1} \cdot$ Anoop Saraya $^{1} \cdot$ Soumya Mahapatra ${ }^{1} \cdot$ Govind Makharia $^{1}$. \\ Anjan Trikha ${ }^{3} \cdot$ Pramod Garg $^{1}$
}

Received: 24 June 2020 / Accepted: 8 July 2020 / Published online: 15 August 2020

(C) Indian Society of Gastroenterology 2020

\begin{abstract}
Background and Aim There is a paucity of data on the clinical presentations and outcomes of Corona Virus Disease-19 (COVID19) in patients with underlying liver disease. We aimed to summarize the presentations and outcomes of COVID-19-positive patients and compare with historical controls.

Methods Patients with known chronic liver disease who presented with superimposed COVID-19 $(n=28)$ between 22 April 2020 and 22 June 2020 were studied. Seventy-eight cirrhotic patients without COVID-19 were included as historical controls for comparison.

Results A total of 28 COVID-19 patients (two without cirrhosis, one with compensated cirrhosis, sixteen with acute decompensation $[\mathrm{AD}]$, and nine with acute-on-chronic liver failure [ACLF]) were included. The etiology of cirrhosis was alcohol $(n=9)$, non-alcoholic fatty liver disease $(n=2)$, viral $(n=5)$, autoimmune hepatitis $(n=4)$, and cryptogenic cirrhosis $(n=6)$. The clinical presentations included complications of cirrhosis in $12(46.2 \%)$, respiratory symptoms in $3(11.5 \%)$, and combined complications of cirrhosis and respiratory symptoms in $11(42.3 \%)$ patients. The median hospital stay was 8 (7-12) days. The mortality rate in COVID-19 patients was 42.3\% (11/26), as compared with $23.1 \%(18 / 78)$ in the historical controls $(p=0.077)$. All COVID-19 patients with ACLF $(9 / 9)$ died compared with $53.3 \%(16 / 30)$ in ACLF of historical controls $(p=0.015)$. Mortality rate was higher in COVID-19 patients with compensated cirrhosis and $\mathrm{AD}$ as compared with historical controls $2 / 17$ (11.8\%) vs. 2/48 (4.2\%), though not statistically significant $(p=0.278)$. Requirement of mechanical ventilation independently predicted mortality (hazard ratio 13.68). Both non-cirrhotic patients presented with respiratory symptoms and recovered uneventfully.

Conclusion COVID-19 is associated with poor outcomes in patients with cirrhosis, with worst survival rates in ACLF. Mechanical ventilation is associated with a poor outcome.
\end{abstract}

Keywords Acute-on-chronic liver failure - Acute decompensation · Chronic liver disease - COVID-19 - Hepatic failure · Inflammation $\cdot$ Liver $\cdot$ Liver function tests $\cdot$ Pandemic $\cdot$ SARS-CoV-2

Shalimar

drshalimar@gmail.com

1 Department of Gastroenterology and Human Nutrition, All India Institute of Medical Sciences, New Delhi 110 029, India

2 Department of Gastroenterology, All India Institute of Medical Sciences, Patna 801 507, India

3 Department of Anaesthesiology, Pain Medicine and Critical Care, All India Institute of Medical Sciences, New Delhi 110 029, India
4 Department of Medicine, All India Institute of Medical Sciences, New Delhi 110 029, India

5 Department of Microbiology, All India Institute of Medical Sciences, New Delhi 110 029, India

6 Department of Radiodiagnosis, Institute Rotary Cancer Hospital, All India Institute of Medical Sciences, New Delhi 110 029, India

7 Department of Pulmonary Medicine and Sleep Disorders, All India Institute of Medical Sciences, New Delhi 110 029, India

8 Department of Biochemistry, All India Institute of Medical Sciences, New Delhi 110 029, India 


\section{Bullet points of the study highlights}

\section{What is already known?}

- Corona Virus Disease-19 (COVID-19) has a high mortality in patients with comorbidities such as diabetes, hypertension and obesity.

- The effect and outcome of COVID-19 in patients with underlying cirrhosis is unclear.

\section{What is new in this study?}

- Patients with cirrhosis and COVID-19 have poor outcome as compared with those without COVID-19.

- The survival rates are worst among COVID patients with acute-on-chronic liver failure.

\section{What are the future clinical and research implications of the study findings?}

- Future studies should assess the role of newer drugs in patients with cirrhosis.

\section{Introduction}

Since 30 January 2020, when India reported its first case of Corona Virus Disease-19 (COVID-19) caused by the novel Severe Acute Respiratory Syndrome-Corona Virus 2 (SARSCoV-2), the total number of cases have increased to $4,40,462$ as of 22 June 2020 [1]. Although the COVID-19 predominantly presents as a respiratory illness in most patients, extrapulmonary manifestations also have been described [2]. The tropism of the virus to angiotensin-converting enzyme-2 (ACE-2) receptors [3] and its presence on the hepatic endothelial cells and cholangiocytes [4] may predispose to direct hepatotoxic injury [5].

Liver enzyme derangements are common, seen in $14 \%$ to $53 \%$ of COVID-19 cases [2]. The clinical consequences are not well known in patients without liver disease since the majority of these derangements are mild in nature and hence clinically inconsequential [6]. However, their impact on patients with underlying liver diseases is beginning to emerge. Evolving data from a global registry suggest a poor outcome in patients with cirrhosis. Among these, $45 \%$ presented with new decompensation and the mortality rate was $33 \%$. In contrast, 303 patients with chronic liver disease (CLD) without cirrhosis fared better with a mortality rate of $8 \%$ [7].

Genetic variability across the world can potentially impact the severity of SARS-CoV-2 infection, which accounts for a myriad of possible presentations and outcomes. However, there is a paucity of data on the presentations and outcomes of COVID-19 patients with underlying liver diseases from different parts of the world. We report our experience of patients with liver disease and COVID-19.

\section{Methods}

\section{Patient population}

In this observation study, all consecutive patients with known liver disease presenting between 22 April 2020 and 22 June 2020 were included. Patients were diagnosed as COVID-19 based on oro-/naso-pharyngeal SARS-CoV-2 ribonucleic acid (RNA) positive result on reverse transcription polymerase chain reaction (RT-PCR) as per the Indian Council of Medical Research (ICMR) criteria [8]. Data were collected from a prospectively maintained database of all patients from admission until endpoint of the study (discharge or death or deadline of 22 June 2020).

We compared the results of COVID-19-positive cirrhosis patients with age-, sex-, and severity-matched cirrhosis patients without COVID-19 (historical controls) from previously published data from our center $[9,10]$.

\section{Patient evaluation and treatment}

All consecutive patients with liver disease presenting to the emergency services with either fever or respiratory symptoms or chest X-ray showing infiltrates were tested for COVID-19 as per the hospital policy. Patients who had no symptom or tested negative initially were offered to retesting based on the development of new respiratory symptoms or fever at the discretion of the treating physician. Nasal and throat swabs were taken and transported in a viral transport media (Hank's balanced salt solution) and tested by RT-PCR. Patients with confirmed COVID19 pneumonia were offered broad-spectrum antibiotics, hydroxychloroquine (HCQ) with or without ivermectin 
according to the evolving consensus [11]. Complications of liver disease including organ failures were managed according to the standard guidelines [12].

\section{Definitions}

Abnormalities in the liver function tests (LFTs) were based on our hospital cut-off values - bilirubin ( $>1 \mathrm{mg} / \mathrm{dL}$ ), aspartate aminotransferase (AST $>40 \mathrm{IU} / \mathrm{L}$ ), alanine aminotransferase $(\mathrm{ALT}>40 \mathrm{IU} / \mathrm{L})$, and alkaline phosphatase (ALP > $280 \mathrm{IU} / \mathrm{L})$. Cirrhosis was defined by the presence of a nodular outline of liver on imaging and concomitant evidence of portal hypertension, such as a dilated portal vein and splenomegaly. Patients with cirrhosis who had a current history of variceal bleed, ascites, hepatic encephalopathy, or jaundice were labeled as having acute decompensation (AD). Acute-onchronic liver failure (ACLF) was defined according to the European Association the Study of the Liver (EASL)-chronic liver failure (CLIF) consortium definition - characterized by a hepatic or extrahepatic insult in a patient with underlying CLD and associated with prespecified organ failure [13]. The clinical presentations were defined as complications of cirrhosis as mentioned above and as respiratory when the presentation was predominantly with cough, breathlessness, and pneumonia. The hospital stay was counted onwards from the date of COVID-19 diagnosis for cases diagnosed during their hospitalization and from the date of admission for those who presented with a positive test. The clinical severity of COVID19 was defined according to the Ministry of Health and Family Welfare (MOHFW) criteria as follows - mild disease as patients with only upper respiratory tract symptoms without any signs of breathlessness and hypoxia. Moderate severity was defined as the presence of pneumonia with the respiratory rate (RR) between 24 and 30/min and $\mathrm{SpO}_{2}$ between $90 \%$ and $94 \%$ on room air while the severe disease was defined by the presence of pneumonia with $\mathrm{RR}>30 /$ min or $\mathrm{SpO}_{2}<90 \%$ on room air or severe respiratory distress [14].

\section{Statistical analysis}

Normally distributed data were expressed as mean \pm standard deviation (SD), and skewed data were expressed as median with interquartile range. Nominal data were expressed as frequency and percentage. Student's $t$ test or Mann-Whitney $U$ test was used for comparing continuous data as appropriate. The Chi-square test or Fisher's exact test was used for categorical variables as applicable. Univariate and multivariate Cox-regression analysis was performed for analysis of predictors of in-hospital mortality. A $p$-value of $<0.05$ was considered significant. Data were analyzed using IBM SPSS statistical software (Version 20.0, Chicago, IL, USA).

\section{Ethical clearance}

In this retrospective analysis requirement of consent was waived off. The study was approved by the institutional ethics committee.

\section{Results}

A total of 750 COVID-19 positive patients were admitted during the study period. Of these $28(3.7 \%)$ patients had an underlying liver disease- 26 patients had liver cirrhosis, one each had nonalcoholic fatty liver disease (NAFLD) and extrahepatic portal venous obstruction (EHPVO). Among patients with cirrhosis, one had compensated cirrhosis, 16 had AD, and 9 presented with ACLF. The median age of patients was 48 (38-58) years, and 20 $(71.4 \%)$ were males. The etiology of cirrhosis was alcoholrelated in $9(34.6 \%)$, NAFLD in $2(7.7 \%)$, hepatitis B virus (HBV) in $3(11.5 \%)$, hepatitis $\mathrm{C}$ virus (HCV) in $2(7.7 \%)$, autoimmune hepatitis $(\mathrm{AIH})$ in $4(15.4 \%)$, and cryptogenic cirrhosis in $6(23.1 \%)$.

\section{Presentations and outcomes}

\section{Patients without cirrhosis}

Both non-cirrhotic patients, one with NAFLD and other with EHPVO, presented with respiratory symptoms. None of the two developed liver-related complications. The LFTs were normal at admission in both the patients. The patient with EHPVO was pregnant at the time of admission and had gestational diabetes mellitus (GDM). Both these patients recovered uneventfully without the need for oxygen or mechanical ventilation.

\section{Patients with compensated cirrhosis}

One patient had NAFLD-related compensated cirrhosis with baseline Child-Pugh-Turcotte (CTP) score of 5, and a model for end-stage liver disease (MELD) score of 9. His associated comorbidities included diabetes mellitus (DM) and coronary artery disease (CAD). He presented with respiratory symptoms and developed ACLF (grade III) during his hospital stay with respiratory, cardiovascular, and renal failures. The patient succumbed to multiorgan failure on day 4 of hospital admission. COVID-19 was severe in this patient.

\section{Patients with AD}

Sixteen patients had AD. The mean age was $48.6 \pm 10.6$ years, and $12(75 \%)$ were males. The clinical presentations included primarily features of AD in $10(62.5 \%), \mathrm{AD}$ as well as respiratory complications in $5(31.3 \%)$, and respiratory alone in one $(6.3 \%)$. Among the 10 patients presenting with $\mathrm{AD}, 8$ 
had upper gastrointestinal bleeding (UGIB) and 2 had ascites. In the combined presentation, AD included 3 with UGIB and 2 with ascites, and in respiratory 4 had pneumonia and one had upper respiratory tract infection. One patient presented with pneumonia alone and during the hospital stay developed UGIB. Nine $(56.3 \%)$ of these patients had a history of DM. The causes of cirrhosis included alcohol in 6 (37.5\%), HBV, HCV, and NAFLD in $1(6.3 \%)$ each, AIH in $2(12.5 \%)$, and cryptogenic in $5(31.3 \%)$. The LFTs were as follows-bilirubin $1.4 \pm 0.8 \mathrm{mg} / \mathrm{dL}$, AST 50 (37-82) IU/L, ALT 29 (19-42) IU/L, ALP 102 (75299) IU/L, albumin $3.0 \pm 0.8 \mathrm{~g} / \mathrm{dL}$, and international normalized ratio (INR) of $1.3 \pm 0.2$. The mean CTP and MELD scores were $7.2 \pm 1.6$ and $12.1 \pm 3.1$, respectively. At presentation deranged LFT values included bilirubin $(>1 \mathrm{mg} / \mathrm{dL})$ in $10(62.5 \%)$, AST $(>40 \mathrm{IU} / \mathrm{L})$ in 10 $(62.5 \%)$, ALT (>40 IU/L) in $4(25 \%)$, ALP (>280 IU/ L) in $4(25 \%)$. Median hospital stay was 8 (6-17) days in 16 patients with AD. Of these, $15(93.8 \%)$ recovered and were discharged. One patient $(6.3 \%)$ required invasive mechanical ventilation and succumbed to illness after developing grade III ACLF (cardiovascular, respiratory, renal, and cerebral failure). Overall, COVID-19 severity was mild in $14(87.5 \%)$, and moderate and severe in 1 $(6.3 \%)$ each.

\section{Patients with ACLF}

Nine patients had ACLF at presentation; the mean age was $47.4 \pm 13.6$ years, and $6(67 \%)$ were males. Of these 9 patients, grade I, II, and III ACLF were present in 3 (33\%), 2 (22.2\%), and $4(44.4 \%)$, respectively. The causes of cirrhosis included alcohol in 3 (33\%), HBV and AIH in 2 (22\%) each, and HCV and cryptogenic in one (11\%) each. The clinical presentations included complications of cirrhosis along with respiratory complaints in 6 (67\%), complications of cirrhosis alone in $2(22 \%)$, and only respiratory complaints in one patient.

Three (33\%) patients with ACLF had DM. The LFTs were as follows - bilirubin 5.3 (3.4-18.4) mg/dL, AST 75 (61-140) IU/L, ALT 37 (25-59) IU/L, ALP 174 (88-489) IU/L, albu$\min 2.8 \pm 0.7 \mathrm{~g} / \mathrm{dL}$, and INR $2.6 \pm 1.2$.

The mean CTP, MELD score, and chronic liver failureconsortium organ failure (CLIF-C OF) and CLIF-C ACLF scores were $11.1 \pm 0.9,28.4 \pm 7.7,13.8 \pm 2.7$, and $58.0 \pm$ 13.6, respectively. At presentation, deranged bilirubin and AST were seen in all, ALT in 4 (44\%) and alkaline phosphatase in 4 (44\%). Of the 9 patients with ACLF, all died over a median hospital stay of 7 (5-8) days. The cause of death was acute respiratory distress syndrome with multiorgan failure in all $9(100 \%)$ patients. Overall, COVID-19 was severe in 7 (77.8\%), and mild and moderate in $1(11.1 \%)$ patient each.

\section{Comparison of characteristics between cirrhotic patients with COVID-19 and historical controls}

We compared COVID-19 patients with cirrhosis $(n=26)$ with age-, sex-, and severity-matched historical controls $(n=78)$, from a previously published data from our center (Table 1). There was no difference in characteristics between the COVID-19-positive cases and historical controls except platelet count and alkaline phosphatase, which were higher in historical controls.

Overall, the mortality rate was higher in cirrhosis patients with COVID-19 (11/26 [42.3\%] vs. 18/78 [23.1\%]), though the difference just fell short of statistical significance $(p=$ 0.077). The mortality rate among COVID-19 patients presenting as ACLF was $100 \%(9 / 9)$, while that in historical controls with ACLF was $53.3 \%(16 / 30)(p=0.015)$. The mortality rate among COVID-19 patients presenting as compensated cirrhosis and $\mathrm{AD}$ was $11.8 \%(2 / 17)$, compared with $4.2 \%(2 / 48)$ among historical controls $(p=0.278)$. There were no differences between the prognostic scores of ACLF patients with COVID-19 and historical controls-MELD (28.4 \pm 7.7 vs. $30.9 \pm 6.6, p=0.347)$, and CLIF-C ACLF score (58.0 \pm 13.6 vs. $50.8 \pm 10.7, p=0.107)$.

\section{Predictors of mortality}

On univariate analysis, the factors significant for outcome among COVID-19 patients were total leukocyte count (TLC), total bilirubin, creatinine, INR, CTP score, MELD score, and requirement of invasive ventilation (Table 2). On multivariate analysis only mechanical ventilation was independently associated with mortality HR, 13.680 (1.390$134.582, P=0.025)$ after adjusting for MELD, and TLC.

\section{Use of COVID-19 specific drugs}

Twenty-five patients received specific drugs for COVID-19: HCQ 21 (84\%), low molecular weight heparin (LMWH) 5 (20\%), vitamin C 22 (88\%), zinc 10 (40\%), azithromycin 20 $(80 \%)$, ivermectin $13(52 \%)$, and methylprednisolone 5 $(20 \%)$.

\section{Discussion}

In this analysis from a tertiary care center in India, we describe the clinical presentations and outcomes of COVID-19 in patients with underlying liver disease. Over 8 weeks, we included 28 patients with underlying liver disease presenting with COVID-19. A higher number of patients reported in the latter part of the study were in accordance with the increasing incidence of COVID-19 in India [15]. Overall, COVID-19 severity was mild in $15(57.7 \%)$, moderate in $2(7.7 \%)$, and severe 
Table 1 Comparison of patients with cirrhosis and Corona Virus Disease-19 (COVID-19) and historical controls with cirrhosis

\begin{tabular}{|c|c|c|c|}
\hline Characteristics & COVID-19-positive $(n=26)$ & Historical controls-cirrhosis patients $(n=78)$ & $P$-value \\
\hline Mean age (years) & $48.5 \pm 12.1$ & $49.1 \pm 11.7$ & 0.804 \\
\hline Sex, male $n(\%)$ & $19(73.1 \%)$ & $56(71.8 \%)$ & 1.000 \\
\hline $\begin{array}{l}\text { Etiology of cirrhosis } \\
\text { Alcohol } \\
\text { Viral } \\
\text { AIH } \\
\text { Cryptogenic } \\
\text { Others }\end{array}$ & $\begin{array}{l}9(34.6 \%) \\
5(19.2 \%) \\
4(15.4 \%) \\
6(23.1 \%) \\
2(7.7 \%)\end{array}$ & $\begin{array}{l}27(34.6 \%) \\
14(17.9 \%) \\
8(10.3 \%) \\
24(30.8 \%) \\
5(6.4 \%)\end{array}$ & 0.923 \\
\hline $\begin{array}{l}\text { Clinical presentation } \\
\text { Compensated cirrhosis and } \mathrm{AD} \\
\text { ACLF }\end{array}$ & $\begin{array}{l}17(65.4 \%) \\
9(34.6 \%)\end{array}$ & $\begin{array}{l}48(61.5 \%) \\
30(38.5 \%)\end{array}$ & 0.817 \\
\hline ACLF grade (I/II/III) & $3 / 2 / 4$ & $10 / 6 / 14$ & 0.988 \\
\hline Hemoglobin $(g / d L)$ & $8.8 \pm 3.2$ & $8.7 \pm 2.9$ & 0.864 \\
\hline Total leukocyte count (per $\mathrm{mm}^{3}$ ) & $7070(5075-12,900)$ & $8500(4775-10,875)$ & 0.719 \\
\hline Platelet count $\left(\times 10^{3} / \mathrm{mm}^{3}\right)$ & $71.5(47.5-119.2)$ & $101.5(61.5-161.2)$ & 0.020 \\
\hline INR & $1.7 \pm 1.0$ & $1.9 \pm 0.9$ & 0.355 \\
\hline Total bilirubin (mg/dL) & $2.1(1.0-4.2)$ & $2.0(0.9-6.9)$ & 0.747 \\
\hline Creatinine & $1.5 \pm 1.3$ & $1.2 \pm 1.0$ & 0.266 \\
\hline AST (IU/L) & $67(43-108)$ & $63(40-126)$ & 0.867 \\
\hline ALT (IU/L) & $30(22-50)$ & $42(24-78)$ & 0.067 \\
\hline Alk P (IU/L) & $111(85-303)$ & $268(195-406)$ & $<0.001$ \\
\hline Albumin (g/dL) & $2.8 \pm 0.7$ & $3.2 \pm 0.9$ & 0.123 \\
\hline CTP & $8.6 \pm 2.3$ & $9.5 \pm 2.8$ & 0.149 \\
\hline MELD & $18.1 \pm 9.6$ & $19.6 \pm 10.2$ & 0.528 \\
\hline In-hospital mortality (\%) & $11(42.3 \%)$ & $18(23.1 \%)$ & 0.077 \\
\hline
\end{tabular}

$A D$ acute decompensation, $A C L F$ acute-on-chronic liver failure, $A I H$ autoimmune hepatitis, $A L T$ alanine aminotransferase, $A l k P$ alkaline phosphatase, $A S T$ aspartate aminotransferase, CTP Child-Turcotte-Pugh, $H B V$ hepatitis B virus, $H C V$ hepatitis $\mathrm{C}$ virus, INR international normalized ratio, $M E L D$ model for end-stage liver disease

in 9 (34.6\%). We observed that COVID-19 patients with cirrhosis presented with complications of cirrhosis in 12 (46.2\%), respiratory symptoms in $3(11.5 \%)$, and combined complications of cirrhosis and respiratory symptoms in 11 (42.3\%). The high rate of respiratory symptoms is expected as SARS-CoV-2 is transmitted via the respiratory route. Our data suggest that cirrhosis patients presenting with respiratory symptoms and recent onset of decompensation/deterioration of underlying liver disease should be evaluated for SARSCoV-2 infection. Patients with cirrhosis and ACLF are at increased risk of severe COVID-19 because of impaired immune status. We observed patients with compensated cirrhosis deteriorate after COVID-19. Furthermore, none of ACLF patients with concomitant COVID-19 survived in our study, as compared with $47 \%$ survival rate in ACLF patients of historical control. The presence of pneumonia in more than $50 \%$ of patients in the COVID-19 group could have led to a higher proportion of patients developing AD and ACLF. On multivariate analysis, we found requirement of invasive ventilation to be an independent predictor of outcome in COVID-19 patients. The higher mortality rates in COVID-19 patients could possibly be because of extrahepatic organ failures especially respiratory failure. The severity of lung injury and CLIF-C has been associated with poor outcomes in COVID-19 patients with cirrhosis [16]. Pulmonary vascular changes including capillary microthrombi are common in patients with COVID-19 [17]. The exact mechanisms related to poor outcomes need exploration.

The outcome parameters in our study are similar to those reported by a multicenter registry (dated 19 June 2020) (Surveillance Epidemiology of Coronavirus) Under Research Exclusion (SECURE)-CIRRHOSIS registry and EASL supported COVID-Hep [7]. The registry reported the outcome of 833 patients with CLD. Of the 303 patients with CLD without cirrhosis, the requirement of invasive ventilation was $18 \%$ and mortality was $8 \%$. Among the 379 patients with cirrhosis (alcohol 32\%, non-alcoholic steatohepatitis 20\%, viral $18 \%$, and alcohol and HCV combined 5\%), decompensation occurred in $45 \%$, the requirement of invasive ventilation was $19 \%$, and death occurred in 33\%. Among the 151 liver transplant (LT) patients, the requirement of invasive ventilation was 19\% and death occurred in 19\% of patients. These 
Table 2 Univariate analysis of predictors of in-hospital mortality among patients with COVID-19 and cirrhosis of liver

\begin{tabular}{lll}
\hline Variable & $\begin{array}{l}\text { Univariate hazard ratio } \\
\text { (HR) }\end{array}$ & $\begin{array}{l}p \text { - } \\
\text { value }\end{array}$ \\
\hline Mean age (years) & $1.024(0.969-1.083)$ & 0.397 \\
Sex, female $n(\%)$ & $1.277(0.329-4.959)$ & 0.723 \\
Diabetes, present & $0.648(0.183-2.297)$ & 0.501 \\
Hemoglobin $(\mathrm{g} / \mathrm{dL})$ & $1.043(0.874-1.245)$ & 0.637 \\
Total leukocyte count $\left(\mathrm{per}^{3} \mathrm{~mm}^{3}\right)$ & $1.061(0.989-1.138)$ & 0.096 \\
Platelet count $\left(\times 10^{3} / \mathrm{mm}^{3}\right)$ & $1.002(0.996-1.009)$ & 0.468 \\
INR & $1.482(1.000-2.195)$ & 0.050 \\
Total bilirubin $(\mathrm{mg} / \mathrm{dL})$ & $1.060(1.009-1.114)$ & 0.021 \\
Creatinine & $1.504(1.010-2.238)$ & 0.044 \\
AST (IU/L) & $1.005(0.999-1.012)$ & 0.109 \\
ALT (IU/L) & $1.013(.984-1.043)$ & 0.372 \\
Alk P (IU/L) & $1.002(0.999-1.005)$ & 0.188 \\
Albumin $(\mathrm{g} / \mathrm{dL})$ & $0.943(0.404-2.200)$ & 0.891 \\
CTP & $1.680(1.205-2.341)$ & 0.002 \\
MELD & $1.084(1.026-1.146)$ & 0.004 \\
Requirement of invasive & $18.858(2.406-147.809)$ & 0.005 \\
$\quad$ ventilation & & \\
\hline
\end{tabular}

$A L T$ alanine aminotransferase, $A l k P$ alkaline phosphatase, $A S T$ aspartate aminotransferase, CTP Child-Turcotte-Pugh, INR international normalized ratio, $M E L D$ model for end-stage liver disease

results suggest that patients with cirrhosis are at a higher risk of mortality post-COVID-19.

Infections are common in patients with ACLF and are associated with poor outcomes [9]. Whether SARS-CoV-2 infection outcomes are similar to other acute precipitants, including bacterial infection in patients developing ACLF, is unclear. A recent study reported poor outcomes in COVID-19-patients as compared with those with bacterial infection [16]. The definite management of ACLF is LT. The current society guidelines differ in their opinion regarding LT in the current scenario. The American Association for the Study of Liver Disease (AASLD) recommends not to postpone transplants as it is an essential medical service while the EASL and Asian Pacific Association for the Study of the Liver (APASL) recommend restricting transplants to those with poor short-term prognosis [18]. The medical treatment options that have been tried for the management of COVID-19 patients include drugs like hydroxychloroquine, remdesivir, other antivirals, and plasma therapy. None of these therapies has been shown to definitely improve the outcome. Moreover, most of these have hepatic side effects. Therefore, there is an urgent need for effective therapies to improve outcomes. We followed the standard recommendation for the management of all complications. For patients who presented with UGIB, we delayed endoscopy and managed patients with vasoconstrictors like terlipressin and patients were started on carvedilol for secondary prophylaxis [19]. All the patients could be managed medically without an increased risk of rebleeding.
We found that the proportion of patients with COVID-19 with alcoholic etiology of CLD was less as compared with our previous experience [20]. In the preCOVID era, the highest fraction of CLD patients admitted was due to alcohol etiology [9]. Lockdown was implemented by the Indian government, and alcohol was not available; this could be a reason, and another possible reason could be the fact the borders of the states were sealed, which would reduce the number of patients reaching the hospital.

The derangement of LFTs at presentations has been reported in up to $50 \%$ of patients without liver disease [21]. The exact significance of the rise in transaminases and its relation to outcomes is unclear. The rise in liver enzymes could be multifactorial due to drugs side effects, associated ischemic hepatitis, and possibly related to SARS-CoV-2 infection per se or the associated cytokine storm [2].

Limitations of the study include its descriptive nature. Due to a small number of patients, we cannot rule out chances of type II statistical errors. The comparison group was taken from historical control admitted during different time frame. The drug therapies varied as per the evolving guidelines and treating team's consensus. Our center is a tertiary care center; the proportion of patients with advanced liver disease was higher as compared with chronic hepatitis and compensated cirrhosis. Our results need to be validated in larger sample size before generalization.

In conclusion, COVID-19 in patients presenting as ACLF is associated with a poor outcome. Overall, in cirrhotic patients, COVID-19 appears to confer higher mortality as compared with those without it. Mechanical ventilation is associated with a poor outcome. In the absence of definite therapies available for COVID-19, it is imperative to prevent COVID19 infection in patients with cirrhosis.

Acknowledgments We thank the Clinical Research Unit, All India Institute of Medical Sciences, New Delhi. We thank the Department of Emergency Medicine, Department of Anaesthesiology, Pain Medicine and Critical Care and Department of Laboratory Medicine, All India Institute of Medical Sciences, New Delhi.

We thank Dr. Upendra Baitha, Dr. Srikant Mohta, and Dr. Chandan Palle for their contribution in patient management and data collection. We thank Prof. Subrat Kumar Acharya for critically reviewing the manuscript.

Name of the department and institutions where the work was done: Department of Gastroenterology and Human Nutrition and dedicated COVID-19 facility Jai Prakash Narayan Apex Trauma Centre (JPNATC), All India Institute of Medical Sciences, New Delhi, India

Author contributions Shalimar: study design, patient management, data analysis, manuscript drafting, and revision of the manuscript

Anshuman Elhence, Manas Vaishnav, and Piyush Pathak: patient management, data collection, and manuscript drafting

Ramesh Kumar: critical review of the manuscript

Kapil Dev Soni, Richa Aggarwal Manish Soneja, Pankaj Jorwal, Arvind Kumar, Puneet Khanna, Akhil Kant Singh, Ashutosh Biswas, Neeraj Nischal, Lalit Dar, Aashish Choudhary, Krithika Rangarajan, 
Anant Mohan, Pragyan Acharya, Baibaswata Nayak, Deepak Gunjan, Anoop Saraya, Soumya Mahapatra, Govind Makharia, Anjan Trikha and Pramod Garg: patient management, data collection, revision of the manuscript

All authors approved the final version of the manuscript.

\section{Compliance with ethical standards}

Conflict of interest $\mathrm{S}, \mathrm{AE}, \mathrm{MV}, \mathrm{RK}, \mathrm{PP}, \mathrm{KDS}, \mathrm{RA}, \mathrm{MS}, \mathrm{PJ}, \mathrm{AK}, \mathrm{PK}$, AKS, AB, NS, LD, AC, KR, AM, PA, BN, DG, AS, SM, GM, AT, and $\mathrm{PG}$ declare that they have no conflict of interest.

Ethics statement The authors declare that the study was performed in a manner conforming to the Helsinki declaration of 1975, as revised in 2000 and 2008 concerning human and animal rights. The study was carried out after approval from the Institutional Ethics Committee, All India Institute of Medical Sciences, New Delhi (Ref No: IEC 253/ 17.04.2020).

Disclaimer The authors are solely responsible for the data and the content of the paper. In no way, the Honorary Editor-in- Chief, Editorial Board Members, or the printer/publishers are responsible for the results/ findings and content of this article.

\section{References}

1. India's first case of coronavirus confirmed in Kerala [Internet]. Inshorts - Stay Informed. [cited 2020 Jun 10]. Available from: https://inshorts.com/en/news/indias-first-case-of-coronavirusconfirmed-in-kerala-1580372444417

2. Elhence A, Shalimar. COVID-19: beyond respiratory tract. J Dig Endosc. 2020;11:24-6.

3. Hamming I, Timens W, Bulthuis ML, Lely AT, Navis G, van Goor $\mathrm{H}$. Tissue distribution of ACE2 protein, the functional receptor for SARS coronavirus. A first step in understanding SARS pathogenesis. J Pathol. 2004;203:631-7.

4. Chai $\mathrm{X}, \mathrm{Hu} \mathrm{L}$, Zhang $\mathrm{Y}$, et al. Specific ACE2 expression in cholangiocytes may cause liver damage after 2019-nCoV infection. bioRxiv. Cold Spring Harbor Laboratory. 2020;2020(02): 03.931766.

5. Bangash MN, Patel J, Parekh D. COVID-19 and the liver: little cause for concern. Lancet Gastroenterol Hepatol. 2020;5:529-30.

6. Phipps MM, Barraza LH, LaSota ED, et al. Acute liver injury in COVID-19: prevalence and association with clinical outcomes in a large US cohort. Hepatology. 2020. https://doi.org/10.1002/hep. 31404

7. weeklyupdate_20200619_web.pdf [Internet]. [cited 2020 Jun 22]. Available from: https://www.covid-hep.net/img/weeklyupdate 20200619_web.pdf
8. Strategey_for_COVID19_Test_v4_09042020.pdf [Internet]. [cited 2020 Jun 10]. Available from: https://www.icmr.gov.in/pdf/covid/ strategy/Strategey_for_COVID19_Test_v4_09042020.pdf

9. Shalimar, Rout G, Jadaun SS, et al. Prevalence, predictors and impact of bacterial infection in acute on chronic liver failure patients. Dig Liver Dis. 2018;50:1225-31.

10. Rout G, Sharma S, Gunjan D, et al. Development and validation of a novel model for outcomes in patients with cirrhosis and acute variceal bleeding. Dig Dis Sci. 2019;64:2327-37.

11. RevisedNationalClinicalManagementGuidelineforCOVID1931032020.pdf [Internet]. [cited 2020 Jun 10]. Available from: https://www.mohfw.gov.in/pdf/RevisedNationalClinical ManagementGuidelineforCOVID1931032020.pdf

12. European Association for the Study of the Liver. Electronic address: easloffice@easloffice.eu, European Association for the Study of the Liver. EASL Clinical Practice Guidelines for the management of patients with decompensated cirrhosis. J Hepatol. 2018;69:406-60.

13. Moreau R, Jalan R, Gines P, et al. Acute-on-chronic liver failure is a distinct syndrome that develops in patients with acute decompensation of cirrhosis. Gastroenterology. 2013;144:1426-37, 1437.e19.

14. ClinicalManagementProtocolforCOVID19.pdf [Internet]. [cited 2020 Jun 14]. Available from: https://www.mohfw.gov.in/pdf/ ClinicalManagementProtocolforCOVID19.pdf

15. COVID19 Statewise status [Internet]. MyGov.in. 2020 [cited 2020 Jun 12]. Available from: https://mygov.in/corona-data/covid19statewise-status/

16. Iavarone M, D'Ambrosio R, Soria A, et al. High rates of 30-day mortality in patients with cirrhosis and COVID-19. J Hepatol. 2020. https://doi.org/10.1016/j.jhep.2020.06.001.

17. Ackermann M, Verleden SE, Kuehnel M, et al. Pulmonary vascular endothelialitis, thrombosis, and angiogenesis in Covid-19. N Engl J Med. 2020;383:120-8.

18. Lau G, Ward JW. Synthesis of liver associations recommendations for hepatology and liver transplant care during the COVID-19 pandemic. Clin Liver Dis. 2020;15:204-9.

19. Gupta V, Rawat R, Shalimar, Saraya A. Carvedilol versus propranolol effect on hepatic venous pressure gradient at 1 month in patients with index variceal bleed: RCT. Hepatol Int 2017;11:181-7.

20. Shalimar, Kedia S, Mahapatra SJ, et al. Severity and outcome of acute-on-chronic liver failure is dependent on the etiology of acute hepatic insults: analysis of 368 patients. J Clin Gastroenterol. 2017;51:734-41.

21. Cai Q, Huang D, Yu H, et al. COVID-19: abnormal liver function tests. J Hepatol. 2020. https://doi.org/10.1016/j.jhep.2020.04.006.

Publisher's note Springer Nature remains neutral with regard to jurisdictional claims in published maps and institutional affiliations. 